\title{
Listening to Sentences in Noise: Revealing Binaural Hearing Challenges in Patients with Schizophrenia
}

\author{
Noor Alaudin Abdul Wahab",2, Mohd. Normani Zakaria1, Abdul Hamid Abdul Rahman, \\ Dinsuhaimi Sidek ${ }^{4}$, and Suzaily Wahab ${ }^{3} \otimes$ \\ ${ }^{1}$ Audiology Programme, School of Health Sciences, Universiti Sains Malaysia, Kelantan, Malaysia \\ ${ }^{2}$ Audiology Programme, School of Rehabilitation Sciences, Faculty of Health Sciences, Universiti Kebangsaan Malaysia, Kuala Lumpur, Malaysia \\ ${ }^{3}$ Department of Psychiatry, Faculty of Medicine, Universiti Kebangsaan Malaysia, Kuala Lumpur, Malaysia \\ ${ }^{4}$ Department of Otorhinolaryngology, School of Medicine, Universiti Sains Malaysia, Kelantan, Malaysia
}

Objective The present, case-control, study investigates binaural hearing performance in schizophrenia patients towards sentences presented in quiet and noise.

Methods Participants were twenty-one healthy controls and sixteen schizophrenia patients with normal peripheral auditory functions. The binaural hearing was examined in four listening conditions by using the Malay version of hearing in noise test. The syntactically and semantically correct sentences were presented via headphones to the randomly selected subjects. In each condition, the adaptively obtained reception thresholds for speech (RTS) were used to determine RTS noise composite and spatial release from masking.

Results Schizophrenia patients demonstrated significantly higher mean RTS value relative to healthy controls ( $\mathrm{p}=0.018)$. The large effect size found in three listening conditions, i.e., in quiet $(\mathrm{d}=1.07)$, noise right $(\mathrm{d}=0.88)$ and noise composite $(\mathrm{d}=0.90)$ indicates statistically significant difference between the groups. However, noise front and noise left conditions show medium $(\mathrm{d}=0.61)$ and small $(\mathrm{d}=0.50)$ effect size respectively. No statistical difference between groups was noted in regards to spatial release from masking on right $(\mathrm{p}=0.305)$ and left $(\mathrm{p}=0.970)$ ear.

Conclusion The present findings suggest an abnormal unilateral auditory processing in central auditory pathway in schizophrenia patients. Future studies to explore the role of binaural and spatial auditory processing were recommended.

Psychiatry Investig 2017;14(6):786-794

Key Words Schizophrenia, Binaural hearing, Spatial hearing, Auditory processing.

\section{INTRODUCTION}

Schizophrenia, ranked as one of the top ten main causes of disease-related disability in the world, ${ }^{1}$ is a mental illness characterized by abnormalities in the perception or expression of reality. The prevalence rate of schizophrenia is about 1.1 percent of the population over the age of 18 years old. ${ }^{2,3}$ Specifically, the average age of onset is 18 years old in male and 25 years old in female with, rare occurrence in population below

Received: September 4, 2016 Revised: December 23, 2016

Accepted: March 19, 2017 Available online: October 12, 2017

$\triangle$ Correspondence: Suzaily Wahab, MD

Department of Psychiatry, Faculty of Medicine, Universiti Kebangsaan Malaysia, Jalan Yaacob Latif, Bandar Tun Razak, Cheras 56000 Kuala Lumpur, Malaysia

Tel: +603 9145 6142, Fax: +603 9145 6188, E-mail: suzailywhb@yahoo.com

(c) This is an Open Access article distributed under the terms of the Creative Commons Attribution Non-Commercial License (http://creativecommons.org/licenses/bync/4.0) which permits unrestricted non-commercial use, distribution, and reproduction in any medium, provided the original work is properly cited.
10 and over 40 years old. ${ }^{4}$ As this is considered the most critical age in social and vocational development, schizophrenia has both economic and non-economic impact on its sufferers, their family members and society. ${ }^{3,5,6}$ Symptoms of schizophrenia which can be divided into a few domains; the positive symptoms, negative symptoms, mood symptoms, motor symptoms and cognitive symptoms may cause significant disability in life and affect multiple areas such as hygiene, self-care, employment and relationship with other people.

The ability to understand spoken language in degraded environment requires an intact auditory system which, enables the segregation of targeted speech signals from unwanted noise. Deficits in the ability, which is one of the core features in central auditory processing disorder [(C)APD], can be investigated by assessing the function of binaural hearing. [(C)APD], despite the presence of intact peripheral hearing, is an abnormal auditory processing that occurs along central auditory path- 
way, i.e., from brainstem towards higher auditory center in the brain. Thus, [(C)APD] is demonstrated by, but not limited to, deficiencies in sound localization and auditory performance in background noise. Binaural hearing, on the other hand, is the ability to listen with two ears. Thus, to perform in background noise, both binaural hearing and central auditory pathway must function normally.

Schizophrenia, with regards to the deficits in cortical GABAergic system, is also associated with deficits in cognitive functions such as in attention and working memory. ${ }^{8}$ In fact, previous studies have found that the presence of competing noise would affect the patients' attention towards important signals. ${ }^{9} 10$ Therefore, auditory processing investigations using sentences are debatable. In order to avoid the limitations, previous studies have applied basic component of speech such as non-meaningful vowel-consonants as stimuli. ${ }^{11-13}$ In spite of minimizing the limitations, it does not reflect our daily verbal conversation activities. Thus, it is more rationale to assess their auditory performance in a simulated conversational environment, i.e listening to sentences in noise. This would minimize the floor and ceiling effects. ${ }^{14}$ In support, a study suggested that patients with schizophrenia were able to utilize content or voice prime to assist their attention to targeted signals such as speech, even in background noise. ${ }^{15}$ The study was conducted using nonsense sentences, i.e., a syntactically correct but semantically not meaningful, to patients with schizophrenia in quiet and background noise. An investigation of speech perception ability using narrative texts embedded in phonetic noise has also been documented. ${ }^{16}$ In addition, a study by Ross et al. ${ }^{17}$ also suggested that schizophrenia patients demonstrated no deficits in recognizing monosyllabic words in background noise. Another study using Speech-in-Babble Perception test, ${ }^{18}$ however, revealed that patients with first-episode psychosis do have difficulties when listening to targeted signals embedded in noise. ${ }^{19}$ Hence, these reflect that the use of words or sentences in noise should be encouraged when assessing the auditory processing of schizophrenia patients. The current study, despite the presence of other hearing in noise test such as Listening in spatialized noise (LISN) ${ }^{20}$ and speech in noise (SIN) ${ }^{21}$ test, utilized the Malay version of hearing in noise test (HINT) to evaluate the binaural auditory processing in patients with schizophrenia. The reason for using HINT test is because it has been validated into Malay version. As all the selected subjects are familiar with Malay language, therefore, the current study has elected the test as the assessment tool for binaural hearing assessment.

HINT is a validated subjective assessment tool developed to assess an individual's binaural hearing on their speech intelligibility in competing background noise. ${ }^{22}$ The tool, which has been validated in other foreign languages, ${ }^{14,23-25}$ including Ma- lay, ${ }^{26}$ uses syntactically correct and semantically meaningful list of sentences. The materials, consisted of three to five word per sentence, have simple lexical and grammatical characteristics which allow listeners' with cognitive and linguistic limitations to take the test. ${ }^{22}$ Relatively, this provides less workload as compared to when using un-meaningful sentences. The test has been applied on various clinical populations such as in children with learning disabilities and cochlear implant users. ${ }^{27,28}$ It measures, by adaptively varying the signal to noise ratio (SNR), the reception thresholds for speech (RTSs) both in quiet and three noise conditions. ${ }^{29}$ The test allows both sentences and noise to be delivered by either loudspeakers or headphones. The use of headphones, which are pre-calibrated to simulate the presentation via loudspeakers, is more preferable as it eliminates the acoustic effect of the environments such as acoustic reverberation. ${ }^{22}$

Spatial release from masking (SRM) refers to a condition when noise, initially co-located with speech signals, is separated from the signals. If the target speech and noise is coming directly from the same direction (e.g., 0-degree azimuth) an individual need a high level of target signal speech to achieve an appropriate signal to noise ratio. On the other hand, when both target speech signal and noise is separated from different direction, there is a release of masking at the amount of $12 \mathrm{~dB}$. This resulting to a better listening condition as compared to both signal and noise coming from the same direction. It provides more realistic speech intelligibility performance in noise. ${ }^{22} \mathrm{~A}$ positive SRM value indicates improved speech performance in noise. ${ }^{29}$ Individuals with intact central auditory processing should show larger SRM value which suggest binaural hearing advantage. ${ }^{30}$ Separating noise from targeted speech signals creates spatial cues, i.e., inter-aural time difference (ITD) and inter-aural level difference (ILD). The cues play important roles when listening and localizing targeted signals in background noise. ${ }^{29}$ Humans' auditory system, from brain stem towards auditory cortex, is sensitive to these cues. Maturity at medial and lateral superior olivary complexes (MSO and LSO) is believed to be the primary stages for binaural unmasking, spatial release from masking and auditory stream segregation. ${ }^{31}$ In addition, inferior colliculus (IC) located in the brain stem is also crucial in binaural and spatial hearing. ${ }^{32}$ The neurons at these locations are sensitive to the phase differences and therefore would be able to improve the SNR following the SRM. ${ }^{33,34}$ Litovsky et al., ${ }^{32}$ argued that, leaving SRM unaffected, unilateral pathology at brain stem level would affect listening performance in noise. The finding suggested that, as part of auditory inputs would bypass IC, SRM should remain unimpaired on either unilateral or contra lateral hemisphere. The only aspects of speech intelligibility in various SNR reported among patients with schizophrenia have been associated with their speech 
recognition or perception ability. ${ }^{15} \mathrm{SRM}$, in addition to adaptive SNR, is related to speech intelligibility due to the relocation of noise source relative to speech signal. In another population of mental disorders, such as attention deficit hyperactivity disorder (ADHD), found impaired focus and failure to suppress relevant signals ${ }^{35}$ and irrelevant distractions respectively. ${ }^{36}$ Study by Mishra et al., ${ }^{37}$ among ADHD children revealed that auditory training by manipulating SNR would improve ADHD symptoms. Hence, as patients with schizophrenia were also associated with attention deficits in noise, further research on SRM among schizophrenic patients are warranted. At this point, no studies have reported the findings of SRM on patients with schizophrenia. Hence, assessments on listening ability in noise and SRM could contribute valuable information to the current knowledge of their auditory processing ability.

\section{METHODS}

\section{Participants}

The present case-control study is part of the work which has been published earlier. ${ }^{38} \mathrm{~A}$ total of 37 right handed participants, i.e 16 schizophrenia patients as cases and 21 healthy controls, were randomly selected. Written consent was obtained from the patients prior to audiological assessments. Ethical approval was obtained from Universiti Kebangsaan Malaysia Medical Centre (UKMMC) ethics committee. The inclusion criteria for both cases and controls include normal hearing and demonstrate good intelligibility in Malay spoken language. As for cases, additional inclusion criteria include being clinically stable with Brief Psychotic Rating Scales (BPRS) less than 31, ${ }^{39}$ and able to fully cooperate during assessments. Cases with extra pyramidal symptoms, neurological disorders and severe psychotic conditions were excluded. The schizophrenic patients which consist of 10 males and 6 females, aged between 19-44 years old were recruited from psychiatry clinic Universiti Kebangsaan Malaysia Medical Centre (UKMMC). The healthy controls, aged between 22-36 years old, consist of 12 males and 9 females. The diagnosis of schizophrenia and the severity of auditory hallucination were determined by the psychiatrists involved in the study, following the DSM-IV criteria and the validated Malay version of Psychotic Symptom Rating Scales (MyPSYRATS) respectively. The MyPSYRATS had shown good internal consistency $(\mathrm{r}=0.85)$ and inter-rater agreement (ICC= $0.877) .{ }^{26}$ All patients at the time of assessments were on atypical antipsychotics.

\section{Audiological procedures}

All subjects underwent audiological procedures in an $18^{\prime} \times$ 12', 40 \pm 2 dBA measured ambient noise level, sound proof room. The procedures consisted ofotoscopic examination, pure tone audiometry, tympanometry, acoustic reflexes, transient otoacoustic emissions and hearing in noise test. Pure tone air conduction thresholds, measured at octave frequency between $250 \mathrm{~Hz}$ and $8000 \mathrm{~Hz}$, were obtained separately for right and left ears by using a Grason-Stadler GSI-61 clinical audiometer plugged with TDH-50P headphones. The purpose of conducting pure tone audiometry was to measure the hearing level and hence determine the hearing status of the subjects. All subjects gave full cooperation and demonstrated good testretest reliability. Normal hearing was defined as thresholds at $20 \mathrm{~dB}$ HL or better across all the frequencies tested.

A Grason-Stadler GSI Tympstar middle ear analyzer was used to assess the middle ear function. Initially, a $226 \mathrm{~Hz}$ probe tone tympanometry with pump speed at $200 \mathrm{daPa} / \mathrm{sec}$ and air pressure from +200 to -400 daPa were performed. A normal Type A tympanogram was characterized as a sharp amplitude peak centered in between $\pm 50 \mathrm{daPa}$. Later, with initial intensity at $75 \mathrm{~dB} \mathrm{HL}$, ipsilateral and contralateral acoustic reflex thresholds were determined by ascending the intensity level in $5-\mathrm{dB}$ steps. The thresholds for acoustic reflexes at 500, $1000 \mathrm{~Hz}$, and $2000 \mathrm{~Hz}$ were defined as the lowest level needed to elicit at least a $0.02 \mathrm{mmhos}$ deflection in middle ear admittance. The presence of type A tympanogram and normal level of acoustic reflexes ( $\leq 95 \mathrm{~dB}$ HL) defines normal middle ear function.

An irregular TEOAE finding among schizophrenic patients have been reported where the amplitude of right is higher than the left ear. ${ }^{40}$ The current study, in order to justify if any abnormalities that might exist in the experiment are not contributed by TEOAE amplitude irregularities, have incorporated TEO$\mathrm{AE}$ with the standard audiological tests mentioned earlier. An Otodynamics analyzer (Otodynamics, Echoport ILO 288) and ILOV6 software package, which generate test stimuli and record TEOAE responses respectively, were used to test the outer hair cells (OHCs) cochlear function. A non-linear click stimulus at $80 \mathrm{dBpe}$ SPL was separately delivered into both ears to determine the functionality of cochlear OHCs. The TEOAE recordings were terminated at 260 sweeps and accepted when the TEOAE responses and stimulus stability was at least $60 \%$ and $80 \%$ respectively. Robust emissions with at least $3 \mathrm{~dB}$ above noise floor suggest healthy OHCs cochlear function.

All subjects, who demonstrated fluent speaking skills in Malay language, underwent the Malay version of HINT. The test is the main tool for research purposes, i.e., to examine the subjects' binaural hearing performance. The test was conducted by a tester who is a native Malay speaker. Prior to the test, without using the headphones, each participant was screened by repeating five randomly selected HINT sentences delivered in normal conversation mode. Each sentence consists of three to five, i.e., six to nine syllables words. Participants who were able to repeat all the sentences correctly were then 
proceed for the actual test. Each subject was tested with 4 20 -sentence lists randomly selected from a total of 12 lists of HINT. In actual test, conducted initially in quiet condition, all subjects were listening to the sentences under headphones. Subsequently, in all three noise conditions, a $65 \mathrm{dBA}$ speech spectrum masking noise was delivered simultaneously with the sentences. The three noise conditions include noise front (i.e., sentences and stimuli were collocated at 0 degree azimuth in front of the listener); noise right (i.e., noise was separated at +90 degree from the sentences) and noise left (i.e., noise was separated at -90 degree from the sentences). Only one list was randomly presented in each condition. The HINT adaptive procedure initially applied $4 \mathrm{~dB}$ steps for adjusting the presentation level of the first 4 sentences. Later, 2 $\mathrm{dB}$ steps were used where the RTS was estimated by averaging the presentation level of the remaining sentences. The adaptive procedure was repeated for each HINT condition. Responses were considered correct if the listener could repeat all the words presented in the sentence without changing the meaning of original sentences. ${ }^{41}$ For instance, adding prefix or suffix in a word without changing the meaning of the sentence is considered as a correct response. On the other hand, SRM was computed by finding the difference between RTS in front and side. The right and left SRM is computed as $\left(\mathrm{RTS}_{\text {front }}{ }^{-}\right.$ $\left.\mathrm{RTS}_{\text {right }}\right)$ and ( $\left.\mathrm{RTS}_{\text {front }}-\mathrm{RTS}_{\text {left }}\right)$ respectively.

\section{Data analysis}

The continuous dependent variables, i.e., RTS and SRM, were measured in both healthy controls and schizophrenic patients. The RTS values were obtained in 4 listening conditions, i.e., in quiet, noise front, noise right and noise left, whilst RTS in noise composite was automatically calculated by the HINT software. Subsequently, the SRM for each ear was determined by calculating the $\mathrm{dB}$ SN difference between RTS noise front relative to RTS noise right and RTS noise left respectively. All data were analyzed using the General Linear Model multivariate analysis in IBM SPSS Statistics for Windows, version 22.0 (IBM Corp., Armonk, NY, USA).

\section{RESULTS}

Table 1 shows the demographic data, mean scores of MyPSYRATS and BPRS in male and female patients with schizophrenia.

In general, with regards to binaural hearing performance, Wilk's Lambda revealed a statistically significant different between the diagnostic groups $[\mathrm{F}(1,35)=3.241, \mathrm{p}=0.018]$. Specifically, the mean of MyHINT scores in four listening conditions, including noise composite and SRM, between the groups were analyzed with one-way MANOVA (Table 2). Statistically significant different $(\mathrm{p}<0.05)$ was found in quiet, noise right and
Table 1. The mean of age, mean scores of MyPSYRATS and BPRS of male and female schizophrenia patients

\begin{tabular}{lcc}
\hline \multicolumn{1}{c}{ Item } & $\begin{array}{c}\text { Male }(\mathrm{N}=10) \\
\text { Mean }(\mathrm{SD})\end{array}$ & $\begin{array}{c}\text { Female }(\mathrm{N}=6) \\
\text { Mean }(\mathrm{SD})\end{array}$ \\
\hline Age & $31.70(6.52)$ & $33.50(8.94)$ \\
MyPSYRATS scores & & \\
Frequency & $1.60(1.84)$ & $1.50(1.98)$ \\
Duration & $1.60(1.65)$ & $1.67(1.97)$ \\
Location & $1.10(1.20)$ & $1.00(1.10)$ \\
Loudness & $1.10(1.40)$ & $1.50(2.0)$ \\
Origin of voice & $1.70(2.00)$ & $1.83(2.04)$ \\
Amount of negative content & $1.40(1.90)$ & $1.17(1.60)$ \\
Degree of negative content & $0.90(1.37)$ & $1.0(1.55)$ \\
Amount of distress & $1.30(1.77)$ & $0.83(1.60)$ \\
Intensity of distress & $1.40(1.90)$ & $0.67(1.20)$ \\
Disruption to life & $0.70(1.34)$ & $0.83(1.6)$ \\
Controllability & $2.30(2.00)$ & $2.00(2.20)$ \\
Total & $15.10(14.66)$ & $14.00(17.15)$ \\
Total BPRS & $8.60(5.95)$ & $7.33(3.72)$ \\
\hline
\end{tabular}

SD: standard deviation, MyPSYRATS: The Malay version of Psychotic Symptom Rating Scales, BPRS: Brief Psychiatric Rating Scale

noise composite with each condition shows a large effect size ( $>0.8$ ). In noise front condition, the $\mathrm{p}$ value was at a brisk of significance ( $\mathrm{p}=0.076$ ), with the effect size ranged from medium to large effect $(\mathrm{d}=0.61)$.

Table 3 compares the data obtained in the current study with the $95 \%$ confidence interval (CI) of Malay version HINT in all listening conditions from normal local populations. ${ }^{41}$ The normative data on SRM were however not available. The comparison, in each condition, reveals the percentage of subjects whom data are not within the normative range. 1) None of healthy controls and $31.3 \%$ schizophrenic patients fail in quiet, 2) $4.8 \%$ healthy controls and $25.0 \%$ schizophrenic patients fail in noise front, 3) $23.8 \%$ healthy controls and $56.3 \%$ schizophrenic patients fail in noise right, 4) $19.0 \%$ healthy controls and $31.3 \%$ schizophrenic patients fail in noise left, and 5) 4.8\% healthy controls and 50.0\% schizophrenic patients fail in noise composite.

\section{DISCUSSION}

The aim of the current study is to investigate the binaural and spatial hearing abilities among schizophrenia patients presented with normal peripheral hearing by using literally, semantically and syntactically correct Malay sentences. To the best of our knowledge, this is the first study that documented such findings.

The first issue that worth discussing is whether sentences- 
Table 2. Statistical analysis of HINT by one way MANOVA

\begin{tabular}{|c|c|c|c|c|c|c|}
\hline \multirow[b]{2}{*}{ HINT variables } & \multicolumn{6}{|c|}{ HINT } \\
\hline & $\begin{array}{c}\text { Normal }(\mathrm{N}=21) \\
\text { Mean } \pm \text { SD }(95 \% \mathrm{CI})\end{array}$ & $\begin{array}{c}\text { Schizophrenia }(\mathrm{N}=16) \\
\text { Mean } \pm \text { SD }(95 \% \mathrm{CI})\end{array}$ & $\mathrm{p}$ value & $\begin{array}{c}95 \% \text { CI for } \\
\text { difference in mean }\end{array}$ & $\begin{array}{l}\text { Cohens'd } \\
\text { effect size }\end{array}$ & $\begin{array}{l}95 \% \text { CI for } \\
\text { effect size }\end{array}$ \\
\hline Quiet & $\begin{array}{c}21.786 \pm 2.394 \\
(20.479-23.092)\end{array}$ & $\begin{array}{c}24.950 \pm 3.557 \\
(23.453-26.447)\end{array}$ & $0.003^{*}$ & $1.18-5.15$ & 1.07 & $0.36-1.74$ \\
\hline Noise front & $\begin{array}{c}-4.495 \pm 0.493 \\
\left(-4.898^{-}-4.092\right)\end{array}$ & $\begin{array}{c}-3.943 \pm 1.268 \\
\left(-4.406^{-}-3.482\right)\end{array}$ & 0.076 & $-0.06-1.16$ & 0.61 & $-0.07-1.26$ \\
\hline Noise right & $\begin{array}{c}-11.666 \pm 0.983 \\
\left(-12.155^{-}-11.178\right)\end{array}$ & $\begin{array}{c}-10.693 \pm 1.243 \\
(-11.253--10.134)\end{array}$ & $0.012^{*}$ & $0.23-1.72$ & 0.88 & $0.18-1.54$ \\
\hline Noise left & $\begin{array}{c}-11.666 \pm 1.012 \\
(-12.142--11.191)\end{array}$ & $\begin{array}{c}-11.131 \pm 1.149 \\
\left(-11.676^{--10.587)}\right.\end{array}$ & 0.142 & $-0.19-1.26$ & 0.50 & $-0.17-1.15$ \\
\hline Noise composite & $\begin{array}{c}-8.071 \pm 0.454 \\
(-8.393--7.750)\end{array}$ & $\begin{array}{c}-7.418 \pm 0.979 \\
(-7.787--7.050)\end{array}$ & $0.010^{*}$ & $0.16-1.14$ & 0.90 & $0.20-1.56$ \\
\hline Right SRM & $\begin{array}{c}7.171 \pm 1.122 \\
(6.631-7.712)\end{array}$ & $\begin{array}{c}6.750 \pm 1.340 \\
(6.131-7.369)\end{array}$ & 0.305 & $-1.24-0.40$ & 0.35 & $-0.99-0.32$ \\
\hline Left SRM & $\begin{array}{c}7.171 \pm 1.087 \\
(6.614-7.729)\end{array}$ & $\begin{array}{c}7.187 \pm 1.455 \\
(6.549-7.826)\end{array}$ & 0.970 & $-0.83-0.86$ & 0.01 & $-0.64-0.66$ \\
\hline
\end{tabular}

*statistically significant at $\mathrm{p}<0.05$. HINT: Hearing In Noise Test, MANOVA: multivariate analysis of variance, SRM: Spatial Release from Masking, SD: standard deviation, CI: confidence interval

based stimuli is appropriate for the patients in the current study. Cognitive issues such as deficits in working memory ${ }^{42}$ and attention ${ }^{43}$ have been documented in schizophrenia patients. Nevertheless, findings on these deficits have been variable. ${ }^{43,44}$ Carter et al..$^{43}$ for instance, argued that attention deficits among schizophrenia patients were more likely due to transient rather than sustained attention. As the current task requires sustained attention, issues regarding the feasibility of the task could be ignored. The current study, as revealed by the patients' mild psychotic condition, ${ }^{39}$ suggest that they are able to cooperate during the assessments. This is supported by their ability to respond appropriately to questions during interview sessions. In addition, during pre-test session, the patients were able to repeat correctly all five HINT sentences presented to them. This suggests that the patients able to proceed with the actual tasks. Hence, depending on the severity of psychosis, we believe that sentences should be encouraged when assessing auditory function in schizophrenia patients. This is consistent with a study done by Lee et al. ${ }^{45}$ that found schizophrenia patients without auditory hallucination demonstrate relatively similar ability with healthy controls when listening to narrative text in low noise conditions. In addition, schizophrenia patients have more impairment in comprehending non-literal compared to literal sentences. ${ }^{46}$ Therefore, as sentences in HINT are syntactically and semantically correct, the test is appropriate for the recruited subjects.

The present findings are consistent with a case-study reported by Litovsky et al. ${ }^{32}$ who conducted audiological assessments on an adult male having small hemorrhage on the right midbrain including the right IC. Despite showing normal periph- eral and spatial hearing, the patient presented with decreased wave $\mathrm{V}$ auditory brainstem (ABR) amplitude following stimulation of the left ear. The patient also complained of experiencing hearing difficulties in background noise. The study suggested that lesion at the brain stem level, in particular IC, contributed to the deficits in binaural hearing and sound localization. In contrast, normal ability in spatial hearing presented by the patient was due to the intact cortical function. Consistently, the present study found that schizophrenia patients required significantly higher mean RTS and performed equally in spatial hearing when compared to healthy controls. In other words, to perform normally in spatial hearing, the patients require louder intensity level. We argued that the problem faced by schizophrenia patients during binaural hearing tasks, specifically hearing in noise, could be contributed by abnormal auditory processing at brainstem level. Our argument is also supported by previous studies on possible brainstem dysfunction in patients with schizophrenia. ${ }^{47-50}$

In addition, based on anatomical and physiological evidences, the involvement of brain stem could be contributed by the widely-reported GABAergic deficiency in patients with schizophrenia. ${ }^{51,52}$ The superior olivary complex (SOC) is consisted with two types of nuclei, i.e., lateral superior olive (LSO) and medial superior olive (MSO). These nuclei, anatomically located at lower brain stem, are positioned on the right and left sides within the brain stem. LSO receives mainly excitatory and inhibitory inputs from ipsilateral and contralateral ventral cochlear nucleus (VCN) respectively. MSO, on the other hand, receives excitatory and inhibitory inputs from both ipsilateral and contralateral VCN. Both LSO and MSO are controlled by 
Table 3. The comparison of RTS of each MyHINT parameters recorded in the subjects of current study with $95 \% \mathrm{Cl}$ value reported by Quar et al. ${ }^{41}$

\begin{tabular}{|c|c|c|c|c|c|c|}
\hline \multirow{2}{*}{$\begin{array}{c}\text { Diagnostic } \\
\text { groups from the } \\
\text { current study }\end{array}$} & \multicolumn{6}{|c|}{ The normative value (95\% CI range) of MyHINT } \\
\hline & Subjects & $\begin{array}{c}\text { Quiet in dB A } \\
\left(13.5^{-26.9)}\right.\end{array}$ & $\begin{array}{c}\text { Noise front in } \mathrm{dB} S / \mathrm{N} \\
\left(-6.4^{-}-3.1\right)\end{array}$ & $\begin{array}{c}\text { Noise right in } \mathrm{dB} S / \mathrm{N} \\
\left(-14.4^{-}-10.4\right)\end{array}$ & $\begin{array}{c}\text { Noise left in dB S/N } \\
(-14.4--10.4)\end{array}$ & $\begin{array}{l}\text { Noise composite in } \\
\mathrm{dB} S / \mathrm{N}\left(-9.8^{--7.3)}\right.\end{array}$ \\
\hline \multirow[t]{11}{*}{ Healthy controls } & N002 & $\mathrm{WNL}$ & WNL & WNL & WNL & WNL \\
\hline & N004 & WNL & WNL & WNL & WNL & WNL \\
\hline & N006 & WNL & WNL & WNL & -10.3 & WNL \\
\hline & N008 & WNL & -3.0 & -10.0 & -8.8 & WNL \\
\hline & N012 & WNL & WNL & -9.3 & WNL & WNL \\
\hline & N014 & WNL & WNL & WNL & -10.2 & WNL \\
\hline & N015 & WNL & WNL & WNL & WNL & WNL \\
\hline & N016 & WNL & WNL & -10.6 & WNL & WNL \\
\hline & N018 & WNL & WNL & -9.7 & WNL & WNL \\
\hline & N021 & WNL & WNL & -10.0 & WNL & WNL \\
\hline & N023 & WNL & WNL & WNL & -9.4 & -7.1 \\
\hline \multirow{14}{*}{$\begin{array}{l}\text { Schizophrenic } \\
\text { patients }\end{array}$} & SCZ001 & WNL & WNL & -9.8 & -10.3 & -6.9 \\
\hline & SCZ002 & WNL & -2.5 & WNL & -7.4 & WNL \\
\hline & SCZ003 & WNL & WNL & -10.3 & WNL & -7.1 \\
\hline & SCZ005 & WNL & WNL & -9.6 & WNL & WNL \\
\hline & SCZ009 & WNL & -2.4 & -9.4 & -8.4 & -5.7 \\
\hline & SCZ012 & WNL & -1.3 & WNL & WNL & -6.1 \\
\hline & SCZ017 & WNL & WNL & -8.9 & WNL & WNL \\
\hline & SCZ 018 & WNL & WNL & -9.8 & -9.6 & -6.7 \\
\hline & SCZ 022 & 29.1 & WNL & -9.6 & WNL & -7.0 \\
\hline & SCZ023 & 27.8 & WNL & -10.3 & WNL & -7.2 \\
\hline & SCZ 024 & 29.2 & -3.0 & -9.8 & -10.3 & -6.5 \\
\hline & SCZ031 & 27.9 & WNL & WNL & WNL & WNL \\
\hline & SCZ032 & WNL & WNL & WNL & WNL & WNL \\
\hline & SCZ 033 & 28.0 & WNL & WNL & WNL & WNL \\
\hline
\end{tabular}

$\mathrm{N}$ : healthy control, SCZ: schizophrenia patients, WNL: within normal limits, RTS: reception thresholds for speech, MyHINT: The Malay version of Hearing In Noise Test, CI: confidence interval. Adapted from Quar et al. Int J Audiol 2008;47:379-380, with permission Taylor \& Francis. ${ }^{41}$

$\mathrm{GABA}_{\mathrm{B}}$ receptors that modulate and maintain the balance in excitation and inhibition of synaptic strength at brain stem level. ${ }^{53}$ We speculate that, due to the GABAergic deficiency in schizophrenia patients, the balance in the synaptic strength is disturbed which eventually affect the binaural hearing performance in general. Specifically, this includes binaural hearing in quiet, in noise and sound localization.

In quiet listening condition, where sentences were delivered to both ears simultaneously, the concept of binaural summation applies. Binaural summation, i.e., the combination of equally presented auditory stimulation from both ears, occurs initially at the level of SOC. ${ }^{54}$ One of the advantages in binaural summation is to improve signal detection. It has been reported that, depending on locations and types of background noise, binaural advantage would improve signal de- tection between 2 and $7 \mathrm{~dB} .{ }^{55}$ The binaural advantage in normal hearing young age adults has been also proven in an electrophysiological study. ${ }^{56}$ Hence, in contrast to monaural listening, binaural advantage allows the perception of auditory signals at softer level. In other words, a person with binaural advantage requires softer incoming signals when listening with two ears. Interestingly, relative to healthy controls, our findings revealed significantly higher mean RTS in patients with schizophrenia. This reflects that in order to perform equally, the schizophrenic patients required louder input signals relative to healthy controls. This infers that binaural summation, specifically binaural advantage, is significantly affected in the patients. Therefore, we speculate that the GABAergicbrain stem deficits in the clinical group might explain the lack of binaural advantage observed in the patients. 
The present study proves that, as found by other researchers, ${ }^{15,45}$ schizophrenic patients do encounter speech in noise deficits. The added value of the present study reveals that the deficits also affect sentences as well. In noise front condition, having a reasonable value of effect size might also indicate that there is a considerable difference between the study groups, even though the $p$ value is insignificant. Effect size is more reliable in statistical decision as it less affected by sample size compared to $\mathrm{p}$ value. ${ }^{57}$ Herein, by considering the effect size value, we concluded that the noise front results between normal and schizophrenia patients were also different. The noise front results obtained in the current study could also agree with Iliadou et al..$^{19}$ whom found patients with early episode of psychosis performed poorer than the healthy controls in majority of central auditory processing tests.

The current study supports, the theory of laterality in schizophrenia patients as revealed by significantly higher mean RTS value in noise-right condition ${ }^{32}$ and better responses when left ear is stimulated by targeted signals. ${ }^{19}$ There are two possibilities that worth discussing. First, LSO and MSO are dominant in processing the information of ILD and ITD respectively. These acoustic cues are important especially in sound localization. In a condition where speech and noise are segregated, for instance in noise right condition, the ILD and ITD of sentences heard on the sentences-dominated-ear will be significantly louder and faster respectively. In other words, the conditions created a sound-localization like task. In healthy controls, the efficiency of $\mathrm{GABA}_{B}$ receptors in modulating the balance in excitatory and inhibitory neurons at brain stem might assist in better sound localization performance especially in background noise. However, due to deficits in GABAergic system in patients with schizophrenia, the imbalance of excitatory and inhibitory could eventually lead to difficulty in sound localization. This was evidenced by the significantly higher mean RTS during noise right condition demonstrated by the schizophrenia patients. Interestingly, our findings revealed that abnormal laterality effect was only observed in noise right instead of noise left condition. Therefore, consistent with Litovsky et al. ${ }^{32}$ we also argue that abnormal laterality in signal processing in schizophrenia patients could begin at least at brainstem level.

Our second argument on abnormal laterality is related to the hemispheres of the brain. When sentences and noise were delivered to the left and right ear respectively, once beyond the SOC, they dominantly travel via its corresponding contralateral afferent auditory pathway towards auditory cortices. ${ }^{54}$ In other words, during noise right condition, speech information and noise travel dominantly via the right and left afferent auditory pathway respectively. Once at the cortical level, rapid and slow speech information is separately processed in the left and right hemispheres respectively. Slow speech information from the right hemisphere was then transferred via corpus collosum towards the left hemisphere for further auditory-related speech processing. Meanwhile, noise that dominantly travelled via the left afferent auditory pathway eventually dominates the left hemisphere. Thus, when the left hemisphere was interrupted by noise, schizophrenia patients demonstrated significantly higher RTS value than healthy controls. The finding supports the deficits of the left hemisphere in schizophrenia. In contrast, when the right hemisphere was dominated by noise during noise left condition, both groups showed no significant difference. This strengthens the presence of abnormal laterality function, especially the left hemisphere, in patients with schizophrenia. This is consistent with previous findings on higher right ear contralateral otoacoustic suppression among schizophrenia patients which suggest abnormal left hemisphere.$^{38}$ However, despite significantly higher mean RTS in noise among patients, the non-significant difference in SRM values between the groups lead us to clinical evidences that worth to be argued.

Litovsky et al..$^{32}$ stated that a reduced unilateral IC function, leaving the spatial hearing unaffected, might disrupt speech intelligibility in noise. As such, they concluded that cortical is more crucial than subcortical regions in mediating spatial hearing abilities. As the current findings are consistent with the mentioned case study, we believe that auditory processing deficits in schizophrenia might even begin at low-level sensory processing. Thus, future studies should focus on combining HINT and objective tests, such as magnetic resonance imaging (MRI), to pinpoint the site of lesions. For instance, studies on healthy controls have shown that the right hemisphere of the brain was more dominant when processing speech signals in noise. ${ }^{58,59}$ Perhaps, by replicating the tests, this could help explain the normal ability of spatial hearing experienced by the schizophrenia patients in the current study. Furthermore, since speech perception deficits in noise is due to impaired binaural hearing, other aspects of binaural hearing (i.e., localization, speech in reverberation, precedence effect, binaural summation) need to be further investigated and documented in these patients.

To the best of our knowledge, this study is the first to unveil the impairment of spatial release from masking in patients with schizophrenia. We acknowledge, as part of limitations, the modest number of sample size collected in the current study. However, based on power and sample size calculations, the power of the current study was up to 0.91 . This suggest that an adequate number of sample size have been recruited in the current study. Hence, despite some limitations as in the modest sample size, the result of the study may still contribute to the current understanding of binaural hearing in pa- 
tients with schizophrenia. In a way, the present study implied that referral to other professionals such as audiologist for binaural hearing training may also be useful. Studies have shown that binaural hearing training is effective in improving speech in noise abilities among patients with asymmetric hearing loss. ${ }^{60}$ In addition, to minimize problem in noise during communication, specific listening tactics can also be applied. For instance, if listening to conversation is problematic when noise is from the left, repositioning the affected ear away from noise is advisable. In addition, listening devices such as FM system could also be recommended.

The benefit of using CIs in reporting clinical findings have been increasingly accepted by researchers. ${ }^{61} \mathrm{~A} 95 \%$ CIs means, if the measure were repeated 100 times, the true value would fall within the interval range 95 times. The current study, despite revealing the statistically small mean group differences, shows clinically meaningful difference in patients with schizophrenia (Table 3). The larger percentage of schizophrenic patients whom HINT in quiet and noise conditions are higher than normative range objectively suggests that schizophrenic patients do have hearing difficulties during binaural listening especially in background noise. In other words, the findings might suggest the existence of auditory processing disorders in patients with schizophrenia. Therefore, the recommendations in providing auditory training rehabilitation among schizophrenic populations is justified.

The current study, in relation to existing neurological theories, supports the evidences on auditory processing deficits among patients with schizophrenia. Difficulties listening in background noise with intact performance in SRM may suggest that the deficits could originate from the brainstem level. Future studies, best aided by imaging technologies, may further help investigate the area of speech processing in noise among patients with schizophrenia.

\section{Acknowledgments}

The research was funded under the Centre for Research and Instrumentation Management (CRIM), Universiti Kebangsaan Malaysia (UKM) grant scheme (GGPM-2012-090).

\section{REFERENCES}

1. WHO. Mental Health Report 2001. Mental Health: New Understanding, New Hope. Geneva: World Health Organization; 2001.

2. Danielyan A, Nasrallah HA. Neurological disorders in Scizophrenia. Psychiatr Clin North Am 2009;32:719-757.

3. Schultz SK, Andreasen NC. Schizophrenia. Lancet 1999;353:1425-1430.

4. Sham PC, MacLean CJ, Kendler KS. A typological model of schizophrenia based on age at onset, sex and familial morbidity. Acta Psychiatr Scand 1994;89:135-141.

5. van Os J, Kapur S. Schizophrenia. Lancet 2009;374:635-645.

6. Tandon R, Nasrallah HA, Keshavan MS. Schizophrenia, "Just the Facts" 5. Treatment and prevention Past, present and future. Schizophr Res 2010;122:1-23.
7. Schminky MM, Baran JA. Central auditory processing disorders: an overview of assessment and managment practices. Deaf Blind Perspect 1999;7:1-16.

8. Vinkers CH, Mirza NR, Olivier B, Kahn RS. The inhibitory GABA system as a therapeutic target for cognitive symptoms in schizophrenia: investigational agents in the pipeline. Expert Opin Investig Drugs 2010; 19:1217-1233.

9. Grillon C, Courchesne E, Ameli R, Geyer MA, Braff DL. Increased distractability in schizophrenic patients. Electrophysiologic and behavioral evidence. Arch Gen Psychiatry 1990;47:171-179.

10. Smucny J, Olincy A, Eichman LC, Lyons E, Tregellas JR. Early sensory processing deficits predict sensitivity to distraction in schizophrenia. Schizophr Res 2013;147:196-200.

11. Hugdahl K, Loberg EM, Jorgensen HA, Lundervold A, Lund A, Green MF, et al. Left hemisphere lateralisation of auditory hallucinations in schizophrenia: a dichotic listening study. Cogn Neuropsychiatry 2008; 13:166-179.

12. Collinson SL, Mackay CE, O J, James AC, Crowb TJ. Dichotic listening impairments in early onset schizophrenia are associated with reduced left temporal lobe volume. Schizophr Res 2009;112:24-31.

13. Hugdahl K, Loberg EM, Falkenberg LE, Johnsen E, Kompus K, Kroken RA, et al. Auditory verbal hallucinations in schizophrenia as aberrant lateralized speech perception: evidence from dichotic listening. Schizophr Res 2012;140:59-64.

14. Vaillancourt V, Laroche C, Mayer C, Basque C, Nali M, Eriks-Brophy A, et al. The Canadian French hearing in noise test. Int J Audiol 2008;47: 383-385.

15. Wu C, Cao S, Zhou F, Wang C, Wu X, Li L. Masking of speech in people with first-episode schizophrenia and people with chronic schizophrenia. Schizophr Res 2012;134:33-41.

16. Lee SH, Chung YC, Yang JC, Kim YK, Suh KY. Abnormal speech perception in schizophrenia with auditory hallucinations. Acta Neuropsychiatr 2004;16:154-159.

17. Ross LA, Saint-Amour D, Leavitt VM, Molholm S, Javitt DC, Foxe JJ. Impaired multisensory processing in schizophrenia: deficits in the visual enhancement of speech comprehension under noisy environmental conditions. Schizophr Res Dec 2007;97:173-183.

18. Iliadou V, Fourakis M, Vakalos A, Hawks JW, Kaprinis G. Bi-syllabic, modern Greek word lists for use in word recognition tests. Int J Audiol 2006;45:74-82.

19. Iliadou VV, Apalla K, Kaprinis S, Nimatoudis I, Kaprinis G, Iacovides A. Is central auditory processing disorder present in psychosis? Am J Audiol 2013;22:201-208.

20. Cameron S, Dillon H, Newall P. The Listening in Spatialized Noise Test: An Auditory Processing Disorder Study. J Am Acad Audiol 2006;17: 306-320.

21. Killion MC, Niquette PA, Gudmundsen GI, Revit LJ, Banerjee S. Development of a quick speech-in-noise test for measuring signal-to-noise ratio loss in normal-hearing and hearing-impaired listeners. J Acoust Soc Am 2004;116:2395-2405.

22. Soli SD, Wong LL. Assessment of speech intelligibility in noise with the hearing in noise test. Int J Audiol 2008;47:356-361.

23. Kronmüller KT, von Bock A, Grupe S, Büche L, Gentner NC, Rückl S, et al. Psychometric evaluation of the Psychotic Symptom Rating Scales. Compr Psychiatry 2011;52:102-108.

24. Gonzalez JC, Sanjuan J, Canete C, Echanove MJ, Leal C. Evaluation of auditory hallucinations: the PSYRATS scale. Actas Esp Psiquiatr 2003; 31:10-17.

25. Favrod J, Rexhaj S, Ferrari P, Bardy S, Hayoz C, Morandi S, et al. French version validation of the psychotic symptom rating scales (PSYRATS) for outpatients with persistent psychotic symptoms. BMC Psychiatry 2012;12:161.

26. Wahab S, Zakaria MN, Sidek D, Abdul Rahman AH, Shah SA, Abdul Wahab NA. Evaluation of auditory hallucinations in patients with schizophrenia: a validation study of the Malay version of Psychotic 
Symptom Rating Scales (PSYRATS). Psychiatry Res 2015;228:462-467.

27. Misurelli SM, Litovsky RY. Spatial release from masking in children with normal hearing and with bilateral cochlear implants: effect of interferer asymmetry. J Acoust Soc Am 2012;132:380-391.

28. Schafer EC. Speech perception in noise measures for children: a critical review and case studies. J Educ Audiol 2010;16:4-15.

29. Litovsky RY. Spatial release from masking. Acoust Today 2012:18-25.

30. Ching TY, van Wanrooy E, Dillon H, Carter L. Spatial release from masking in normal-hearing children and children who use hearing aids. J Acoust Soc Am 2011;129:368-375.

31. Yuen KC, Yuan M. Development of spatial release from masking in Mandarin-speaking children with normal hearing. J Speech Lang Hear Res 2014;57:2005-2023.

32. Litovsky RY, Fligor BJ, Tramo MJ. Functional role of the human inferior colliculus in binaural hearing. Hear Res 2002;165:177-188.

33. Tollin DJ, Yin TC. Interaural phase and level difference sensitivity in low-frequency neurons in the lateral superior olive. J Neurosci 2005;25: 10648-10657.

34. Grothe B, Pecka M, McAlpine D. Mechanisms of sound localization in mammals. Physiol Rev 2010;90:983-1012.

35. Brown TE. ADD/ADHD and impaired executive function in clinical practice. Neurosci Neuropsychol ADHD 2009;1:37-41.

36. Aboitiz F, Ossandon T, Zamorano F, Palma B, Carrasco X. Irrelevant stimulus processing in ADHD: catecholamine dynamics and attentional networks. Front Psychol 2014;5:183.

37. Mishra J, Sagar R, Joseph AA, Gazzaley A, Merzenich MM. Training sensory signal-to-noise resolution in children with ADHD in a global mental health setting. Transl Psychiatry 2016 6:e781.

38. Abdul Wahab NA, Wahab S, Abdul Rahman AH, Sidek D, Zakaria MN. The hyperactivity of efferent auditory system in patients with schizophrenia: a transient evoked otoacoustic emissions study. Psychiatry Investig 2016;13:82-88.

39. Leucht S, Kane JM, Kissling W, Hamann J, Etschel E, Engel R. Clinical implications of Brief Psychiatric Rating Scale scores. Br J Psychiatry 2005; 187:366-371.

40. Veuillet E, Georgieff N, Philibert B, Dalery J, Marie-Cardine M, Collet L. Abnormal peripheral auditory asymmetry in schizophrenia. J Neurol Neurosurg Psychiatry 2001;70:88-94.

41. Quar TK, Mukari SZMS, Abdul Wahab NA, Abdul Razak R, Omar M, Maamor N. The Malay hearing in noise test. Int J Audiol 2008;47:379380 .

42. Seidman LJ, Meyer EC, Giuliano AJ, Breiter HC, Goldstein JM, Kremen WS, et al. Auditory working memory impairments in individuals at familial high risk for schizophrenia. Neuropsychology 2012;26:288-303.

43. Carter JD, Bizzell J, Kim C, Bellion C, Carpenter KL, Dichter G, et al. Attention deficits in schizophrenia-preliminary evidence of dissociable transient and sustained deficits. Schizophr Res 2010;122:104-112.

44. Krieger S, Lis S, Cetin T, Gallhofer B, Meyer-Lindenberg A. Executive Function and Cognitive Subprocesses in First-Episode, Drug-Naive Schizophrenia: An Analysis of N-Back Performance. Am J Psychiatry 2005;162:1206-1208.
45. Lee SH, Chung YC, Yang JC, Kim YK, Suh KY. Abnormal speech perception in schizophrenia with auditory hallucinations. Acta Neuropsychiatr 2004;16:154-159.

46. Pesciarelli F, Gamberoni T, Ferlazzo F, Lo Russo L, Pedrazzi F, Melati E, et al. Is the comprehension of idiomatic sentences indeed impaired in paranoid Schizophrenia? A window into semantic processing deficits. Front Hum Neurosci 2014;8:799.

47. Igata M, Ohta M, Hayashida Y, Abe K. Missing peaks in auditory brainstem responses and negative symptoms in schizophrenia. Jpn J Psychiatry Neurol 1994;48:571-578.

48. Källstrand J, Nehlstedt SF, Sköld ML, Nielzén S. Lateral asymmetry and reduced forward masking effect in early brainstem auditory evoked responses in schizophrenia. Psychiatry Res 2012;196:188-193.

49. Nam EC. Is it necessary to differentiate tinnitus from auditory hallucination in schizophrenic patients? J Laryngol Otol 2005;119:352-355.

50. Tarasenko MA, Swerdlow NR, Makeig S, Braff DL, Light GA. The auditory brainstem response to complex sounds: a potential biomarker for guiding treatment of psychosis. Front Psychiatry 2014;5:142.

51. Kehne JH, Maynard GD. GABA and Schizophrenia. In: Jeffrey S. Albert, Wood MW, Editors. Targets and Emerging Therapies for Shizophrenia. 1 Ed. Hoboken, New Jersey: John Wiley and Sons, Inc, 2012, p.425465.

52. O'Connor WT, O'Shea SD. Clozapine and GABA transmission in schizophrenia disease models: establishing principles to guide treatments. Pharmacol Ther 2015;150:47-80.

53. Grothe B, Koch U. Dynamics of binaural processing in the mammalian sound localization pathway-- the role of GABA(B) receptors. Hear Res 2011;279:43-50.

54. Lazard D, Collette J, Perrot X. Speech processing: from peripheral to hemispheric asymmetry of the auditory system. Laryngoscope 2012; 122:167-173.

55. Hawley ML, Litovsky RY, Culling JF. The benefit of binaural hearing in a cocktail party: effect of location and type of interferer. J Acousti Soc Am 2004;115:833-843.

56. Wong MS. The presence of binaural interaction component (BIC) in the auditory brainstem response (ABR) of normal hearing adults. Doctoral dissertation, Tampa, FL: University of Southern Florida; 2002.

57. Sullivan GM, Feinn R. Using effect size-or why the p value is not enough. J Grad Med Educ 2012;4:279-282.

58. Shtyrov Y, Kujala T, Ilmoniemi RJ, Naatanen R. Noise affects speech-signal processing differently in the cerebral hemispheres. Neuroreport 1999; 10:2189-2192.

59. Shtyrov Y, Kujala T, Ahveninen J, Tervaniemi M, Alku P, Ilmoniemi RJ, Naatanen R. Background acoustic noise and the hemispheric lateralization of speech processing in the human brain: magnetic mismatch negativity study. Neurosci Lett 1998;251:141-144.

60. Constantinides $\mathrm{H}$, Rose MM, Moore DR. Training in binaural hearing: towards its therapeutic use in clinical practice. Int Congr Ser 2003;1254: 481-485.

61. Page P. Beyond statistical significance: clinical interpretation of rehabilitation research literature. Int J Sports Phys Ther 2014;9:726-736. 